\title{
THEORETICAL AND PRACTICAL SPECIFIC ACTIVITIES AND OTHER PROPERTIES OF COMMON RADIONUCLIDES
}

\author{
S. A. Reynolds
}




\section{DISCLAIMER}

This report was prepared as an account of work sponsored by an agency of the United States Government. Neither the United States Government nor any agency Thereof, nor any of their employees, makes any warranty, express or implied, or assumes any legal liability or responsibility for the accuracy, completeness, or usefulness of any information, apparatus, product, or process disclosed, or represents that its use would not infringe privately owned rights. Reference herein to any specific commercial product, process, or service by trade name, trademark, manufacturer, or otherwise does not necessarily constitute or imply its endorsement, recommendation, or favoring by the United States Government or any agency thereof. The views and opinions of authors expressed herein do not necessarily state or reflect those of the United States Government or any agency thereof. 


\section{DISCLAIMER}

Portions of this document may be illegible in electronic image products. Images are produced from the best available original document. 
This report was prepared as an account of work sponsored by the United States Government. Neither the United States nor the United States Atomic Energy Commission, nor any of their employees, nor any of their contractors, subcontractors, or their employees, makes any warranty, express or implied, or assumes any legal liability or responsibility for the accuracy, completeness or usefulness of any information, apparatus, product or process disclosed, or represents that its use would not infringe privately owned rights. 
Contract No. W-7405-eng-26

ISOTOPES DEVELOPMENT CENTER

THEORETICAL AND PRACTICAL SPECIFIC ACTIVITIES AND OTHER PROPERTIES OF COMMON RADIONUCLIDES

S. A. Reynolds :

Isotopes Division

Analytical Chemistry Division

MARCH 1973

NOTICE

This report was prepared as an account of work This report was prepared States Government. Neither the United States nor the United States Ato mic Energy Commission nor any of their employees, nor any of Comission, thej contractors, subeoness implied, or assumes any nrakes any warrans, oxprity for the accuracy, comlegal hability or pleteness or usefulness of any information, apparatus, product or process disclosed, or represen
would not infringe privately owned rights.

NOTICE This document contains information of a preliminary nature and was prepared primarily for internal use at the Oak Ridge National Laboratory. It is subject to revision or correction and therefore does not represent a final report.

\footnotetext{
OAK RIDGE NATIONAL LABORATORY

Oak Ridge, Tennessee 37830 operated by UNION CARBIDE CORPORATION for the U.S. ATOMTC ENERGY CUMMISSIUN
} 


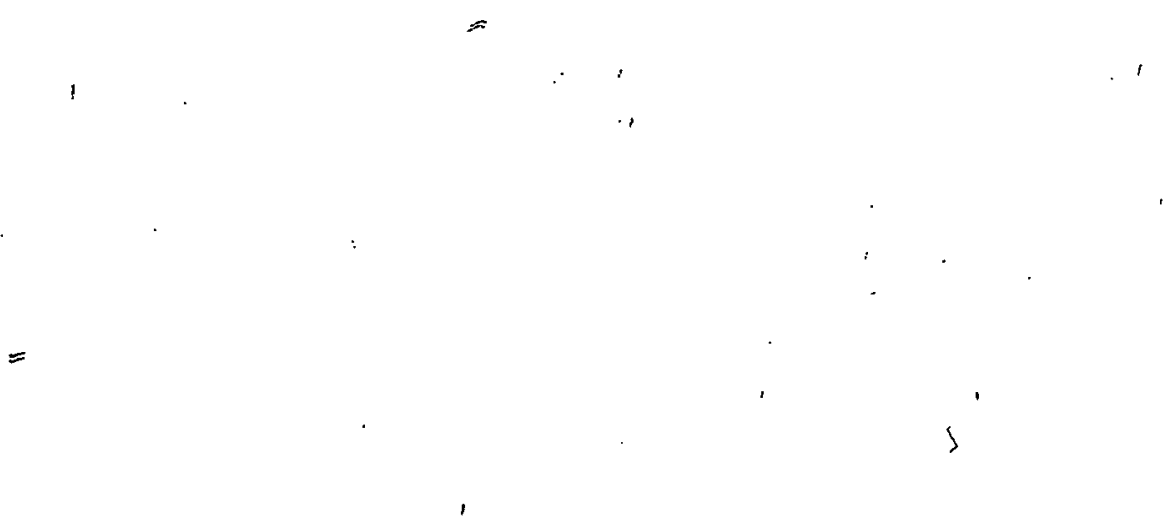

THIS PAGE

WAS INTENTIONALLY

LEFT BLANK 
TABLE OF CONTENTS

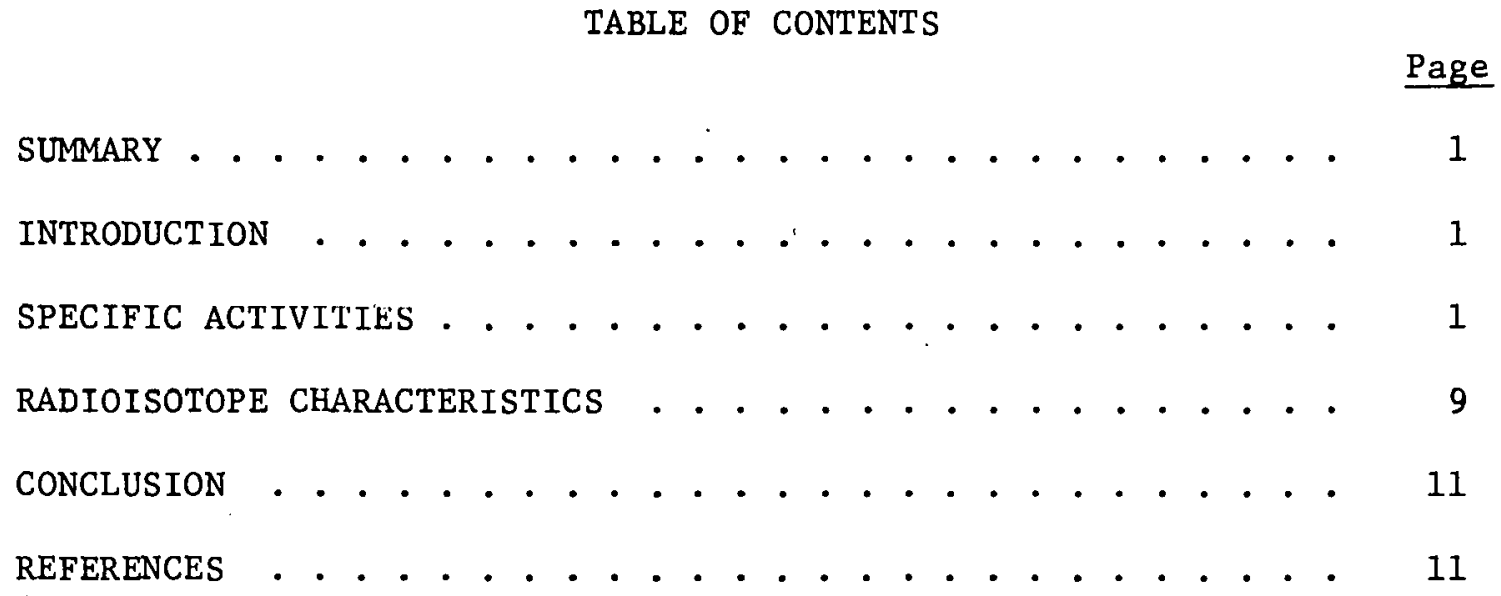


THEORETICAL AND PRACTICAL SPECIFIC ACTIVITIES

AND OTHER PROPERTIES OF COMMON RADIONUCLIDES

\author{
S. A. Reynolds
}

SUMMARY

\begin{abstract}
Half-lives were carefully selected, and specific activities were calculated for 161 commonly used radioisotopes with half-lives greater than $12 \mathrm{hr}$. Practical specific activities of many bombardment products are listed, based where applicable upon critically evaluated neutron cross sections and fission yields, as well as analytical data. Up-to-date decay parameters for a number of additional nuclides are given. The information can be applied by manufacturers and users of radioisotope materials, radiochemical analysts, and others.
\end{abstract}

\title{
INTRODUCTION
}

The radioisotopes program at ORNL has resulted in the production of radionuclides by neutron irradiation, accelerator bombardment, and isolation from natural sources. ${ }^{1}$ Yields, in terms of theoretical and practical specific activities, are listed for many of them. Half-life values and other data serve to improve some data given in the Isotope User's Guide. ${ }^{2}$ This information was originally compiled for use at ORNL and is being published because it may be of use to others who produce or apply radionuclides.

\section{SPECIFIC ACTIVITIES}

The half-life values for the 161 nuclides in Table 1 were taken from the "Radioactive Atoms" table, ${ }^{3}$ issues of Nuclear Data, ORNL work, ${ }^{4}$ and other recent literature. They supersede the selections in the Isotope User's Guide. The isotopic specific activities were calculated as usual. Exponentials are represented in the tables in " $E$ " format, i.e., $k E n$ means $k$ times 10 to the power $n$. The present values replace those tabulated earlier. 5 In many cases, only the theoretical specific activity is listed, since the product is of high abundance because of production by accelerator, $(n, p)$ reaction, or decay of a parent, or because the material is isotopically enriched electromagnetically or by diffusion. Specific activities are useful for calculating curie contents of actinide preparations, for example, for which mass analyses are known. 
Table 1. Theoretical and Practical Specific Activities

\begin{tabular}{|c|c|c|c|c|c|c|}
\hline Nuclide & Half-Life & $\begin{array}{c}\text { Theoretical } \\
\text { Specific } \\
\text { Activity } \\
\text { (Ci/g) }\end{array}$ & $\begin{array}{l}\sigma_{A} \text { of } \\
\text { Target } \\
\text { Nuclide } \\
\text { (barns) }^{a}\end{array}$ & $\begin{array}{l}\sigma_{\mathrm{B}} \text { of } \\
\text { Radio- } \\
\text { nuclide } \\
\text { (barns) }\end{array}$ & $\begin{array}{c}\text { Practical } \\
\text { Specific } \\
\text { Activity } \\
(\mathrm{C} i / \mathrm{g})\end{array}$ & Remarks \\
\hline $26 \mathrm{Al}$ & $7.4 \mathrm{E} 5 \mathrm{y}$ & $1.9 \mathrm{E}-2$ & & & & \\
\hline $241 \mathrm{Am}$ & $433 \cdot y$ & 3.43 & & & $\cdot$ & \\
\hline $243 \mathrm{Am}$ & $7.4 \mathrm{E} 3 \mathrm{y}$ & 0.20 & & & & \\
\hline $122 \mathrm{Sb}$ & $2.7 \mathrm{~d}$ & 4.0E5 & $6.2 \mathrm{R}$ & & $1.4 \mathrm{E} 3$ & $7 \mathrm{~d}$ at $2 \mathrm{E} 15$ \\
\hline $124 \mathrm{Sb}$ & $60.2 \mathrm{~d}$ & $1.75 \mathrm{E} 4$ & $3.4 \mathrm{R}$. & 6.5 & $2.7 \mathrm{E} 2$ & $120 \mathrm{~d}$ at $8 \mathrm{E} 14$ \\
\hline $125 \mathrm{Sb}$ & $2.77 \cdot y$ & $1.03 \mathrm{E} 3$ & & & & \\
\hline $37 \mathrm{Ar}$ & $35 \mathrm{~d}$ & $1.01 \mathrm{E} 5$ & & & & \\
\hline${ }^{39} \mathrm{Ar}$ & $269 y$ & 34 & & , & 2.7 & $8 \%$ abundance \\
\hline 73 As & $80 \mathrm{~d}$ & 2. $2 \mathrm{E} 4$ & & & & \\
\hline${ }^{74} \mathrm{As}$ & $17.8 \mathrm{~d}$ & $9.9 \mathrm{E} 4$ & & & & \\
\hline $76 \mathrm{As}$ & $26.3 \mathrm{~h}$ & $1.6 \mathrm{E} 6$ & 4.3 & & $1.5 \mathrm{E} 3$ & $2.5 \mathrm{~d}$ at $2 \mathrm{E} 15$ \\
\hline 77 As & $38.7 \mathrm{~h}$ & $1.05 \mathrm{E} 6$ & & & & \\
\hline $131 \mathrm{Ba}$ & $12 \mathrm{~d}$ & $8.3 E 4$ & $11 \mathrm{R}$ & & $7.4 \mathrm{E} 2$ & $21 \mathrm{~d}$ at $8 \mathrm{E} 14$ \\
\hline $133 \mathrm{mBa}$ & $38.9 \mathrm{~h}$ & $6.1 \mathrm{E5}$ & & & & \\
\hline $133 \mathrm{Ba}$ & $10.5 \mathrm{y}$ & $2.6 \mathrm{E} 2$ & 8.5 & . & 48 & $1 \mathrm{y}$ at $8 \mathrm{E} 14$ \\
\hline $135 \mathrm{~m}_{\mathrm{Ba}}$ & $28.7 \mathrm{~h}$ & $8.1 \mathrm{E5}$ & & & 26 & $3 \mathrm{~d}$ at $2 \mathrm{E} 15$ (TM-3111) \\
\hline $140 \mathrm{Ba}$ & $12.8 \mathrm{~d}$ & $7.3 \mathrm{E} 4$ & & & 2. $3 \mathrm{E} 4$ & FP, 28-d irradiation \\
\hline $249 \mathrm{Bk}$ & $314 \mathrm{~d}$ & $1.67 \mathrm{E} 3$ & & & & \\
\hline $206 \mathrm{Bi}$ & $6.24 \mathrm{~d}$ & $1.02 \mathrm{E} 5$ & & & & \\
\hline $207 \mathrm{Bi}$ & $30 \mathrm{y}$ & 58 & & & & \\
\hline $210 \mathrm{Bi}$ & $5.0 \mathrm{~d}$ & $1.24 \mathrm{E} 5$ & $0.019 \mathrm{R}$ & & 1.8 & $7 \mathrm{~d}$ at $2 \mathrm{E} 15$ \\
\hline $82 \mathrm{Br}$ & $35.4 \mathrm{~h}$ & $1.08 \mathrm{E} 6$ & $3 \mathrm{R}$ & & $8.4 \mathrm{E} 2$ & $2.5 \mathrm{~d}$ at $2 \mathrm{E} 15$ \\
\hline $109 \mathrm{Cd}$ & $1.26 \mathrm{y}$ & $2.6 \mathrm{E} 3$ & 1.1 & 700 & 4 & Max $130 \mathrm{~d}$ at $8 \mathrm{E} 14$ \\
\hline $115 \mathrm{mCd}$ & $44 d$ & $2.6 \mathrm{E} 4$ & 0.036 & & 3.2 & $90 \mathrm{~d}$ at $8 \mathrm{E} 14$ \\
\hline $115 \mathrm{Cd}$ & $53.5 \mathrm{~h}$ & $5.1 \mathrm{E} 5$ & $0.30 \mathrm{R}$ & & 76 & $7 \mathrm{~d}$ at $2 \mathrm{E} 15$ \\
\hline $41 \mathrm{Ca}$ & $1.3 \mathrm{E} 5 \mathrm{y}$ & $6.7 \mathrm{E}-2$ & & & & \\
\hline${ }^{45} \mathrm{Ca}$ & $164 \mathrm{~d}$ & $1.77 \mathrm{E} 4$ & 0.9 & & 2. $3 \mathrm{E} 2$ & $1 \mathrm{y}$ at $8 \mathrm{E} 14$ \\
\hline${ }^{47} \mathrm{Ca}$ & $4.53 \mathrm{~d}$ & $6.1 \mathrm{E} 5$ & 0.77 & & $3.6 \mathrm{E} 2$ & $7 \mathrm{~d}$ at $2 \mathrm{E} 15$ \\
\hline
\end{tabular}


Table 1. Continued

\begin{tabular}{|c|c|c|c|c|c|c|}
\hline Nuclide & Half-Life & $\begin{array}{c}\text { Theoretical } \\
\text { Specific } \\
\text { Activity } \\
\text { (Ci/g) }\end{array}$ & $\begin{array}{l}\sigma_{A} \text { of } \\
\text { Target } \\
\text { Nuclide } \\
\text { (barns) }\end{array}$ & $\begin{array}{l}\sigma_{\mathrm{B}} \text { of } \\
\text { Radio- } \\
\text { nuclide } \\
\text { (barns) }\end{array}$ & $\begin{array}{c}\text { Practical } \\
\text { Specific } \\
\text { Activity } \\
\text { (Ci/g) }\end{array}$ & Remarks \\
\hline $\begin{array}{l}252 \mathrm{Cf} \\
{ }^{14} \mathrm{C}\end{array}$ & $\begin{array}{l}2.64 \mathrm{y} \\
5730 \mathrm{y}\end{array}$ & $\begin{array}{l}5.37 \mathrm{E} 2 \\
4.46\end{array}$ & & & & . \\
\hline${ }^{141} \mathrm{Ce}$ & 32.5 & $2.9 E 4$ & & & $4.6 \mathrm{E} 3$ & FP, 60-d irradiation \\
\hline $144 \mathrm{Ce}$ & $284 \mathrm{~d}$ & $3.2 \mathrm{E} 3$ & & & $7.2 \mathrm{E} 2$ & $\mathrm{FP}, 1 \mathrm{y}$ \\
\hline $129 \mathrm{Cs}$ & $32.6 \mathrm{~h}$ & $7.4 \mathrm{E} 5$ & & & & \\
\hline $13 l_{\mathrm{Cs}}$ & $9.7 \mathrm{~d}$ & $1.03 \mathrm{E} 5$ & & & & \\
\hline $134 \mathrm{Cs}$ & $2.06 \mathrm{y}$ & $1.30 \mathrm{E} 3$ & $30 \mathrm{R}$ & 134 & $1.8 \mathrm{E} 2$ & $180 \mathrm{~d}$ at $8 \mathrm{E} 14$ \\
\hline $137 \mathrm{Cs}$ & $30 \mathrm{y}$ & & & & 30 & $35 \%{ }^{1} 37 \mathrm{Cs}$ \\
\hline${ }^{36} \mathrm{C} 1$ & 3. $0 \mathrm{E} 5 \mathrm{y}$ & 3. $3 E-2$ & & & $2.3 E-2$ & $69 \%{ }^{36} \mathrm{Cl} \quad(\mathrm{TM}-3677)$ \\
\hline${ }^{51} \mathrm{Cr}$ & $27.7 \mathrm{~d}$ & $9.2 \mathrm{E} 4$ & 16 & & $3.0 \mathrm{E} 3$ & $50 \mathrm{~d}$ at $8 \mathrm{E} 14$ \\
\hline${ }^{56} \mathrm{Co}$ & $77.3 \mathrm{~d}$ & $3.0 \mathrm{E} 4$ & & & & \\
\hline${ }^{57} \mathrm{Co}$ & $271 \mathrm{~d}$ & $8.4 \mathrm{E} 3$ & & & & \\
\hline${ }^{58} \mathrm{Co}$ & $71.3 \mathrm{~d}$ & $3.16 \mathrm{E} 4$ & & & & \\
\hline${ }^{60} \mathrm{Co}$ & $5.27 \mathrm{y}$ & $1.13 \mathrm{E} 3$ & & & $1 \mathrm{E} 3$ & \\
\hline${ }^{64} \mathrm{Cu}$ & $12.7 \mathrm{~h}$ & $3.9 \mathrm{E} 6$ & 4.5 & · & $1.7 \mathrm{E} 3$ & $1 \mathrm{~d}$ at $2 \mathrm{E} 15$ \\
\hline${ }^{67} \mathrm{Cu}$ & $61: 8 \mathrm{~h}$ & $7.6 \mathrm{E5}$ & & & & \\
\hline $242 \mathrm{Cm}$ & $163 \mathrm{~d}$ & $3.32 \mathrm{E} 3$ & & & & \\
\hline $244 \mathrm{Cm}$ & $18.1 \mathrm{y}$ & 80.9 & & & & \\
\hline $248 \mathrm{Cm}$ & $3.5 \mathrm{E5} \mathrm{y}$ & $4 \cdot 1 \mathrm{E}-2$ & & & & \\
\hline $152 \mathrm{Eu}$ & $13 \mathrm{y}$ & $1.8 \mathrm{E} 2$ & $5.3 \mathrm{E} 3$ & $5.3 \mathrm{E} 3$ & 64 & $\mathrm{TM}-3611$ \\
\hline $154 \mathrm{Eu}$ & $8 \mathrm{y}$ & $2.9 \mathrm{E} 2$ & 480 & 1450 & 56 & $\begin{array}{l}\operatorname{Max} 7 \mathrm{~d} \text { at } 2 \mathrm{E} 15 \\
(\mathrm{TM}-3611)\end{array}$ \\
\hline${ }^{156} \mathrm{Eu}$ & $15 \mathrm{~d}$ & $5.6 \mathrm{E} 4$ & & & $4 E 4$ & Calc. from TM-3501 \\
\hline $153 \mathrm{Gd}$ & $241 \mathrm{~d}$ & $3.5 \mathrm{E} 3$ & $1.2 \mathrm{E} 2 \mathrm{R}$ & $1 \mathrm{E} 4$ & 78 & $T M-2632$ \\
\hline${ }^{67} \mathrm{Ga}$ & $78 \mathrm{~h}$ & $6.0 \mathrm{E} 5$ & & & & \\
\hline${ }^{72} \mathrm{Ga}$ & $14.1 \mathrm{~h}$ & $3.1 \mathrm{E} 6$ & 5 & & $1.7 \mathrm{E} 3$ & $1 \mathrm{~d}$ at $2 \mathrm{E} 15$ \\
\hline${ }^{68} \mathrm{Ge}$ & $280 \mathrm{~d}$ & $6.8 \mathrm{E} 3$ & & & & \\
\hline${ }^{71} \mathrm{Ge}$ & $11 \mathrm{~d}$ & $1.7 \mathrm{E} 5$ & 3.5 & & $4.6 \mathrm{E} 2$ & $21 \mathrm{~d}$ at $8 \mathrm{E} 14$ \\
\hline
\end{tabular}


Table 1. Continued

\begin{tabular}{|c|c|c|c|c|c|c|}
\hline Nuclide & Half-Life & $\begin{array}{c}\text { Theoretical } \\
\text { Specific } \\
\text { Activity } \\
(\mathrm{Ci} / \mathrm{g})\end{array}$ & $\begin{array}{l}\mathrm{J}_{\mathrm{A}} \text { of } \\
\text { Target } \\
\text { Nuclide } \\
\text { (barns) }\end{array}$ & $\begin{array}{c}\sigma_{\mathrm{B}} \text { of } \\
\text { Radio- } \\
\text { nuclide } \\
\text { (barns) }\end{array}$ & $\begin{array}{l}\text { Practical } \\
\text { Specific } \\
\text { Activity } \\
(\mathrm{Ci} / \mathrm{g})\end{array}$ & Remarks \\
\hline${ }^{195} \mathrm{Au}$ & $184 \mathrm{~d}$ & $3.6 \mathrm{E} 3$ & . & & & \\
\hline${ }^{198} \mathrm{Au}$ & $2.70 \mathrm{~d}$ & $2.4 \mathrm{E} 5$ & $45(\operatorname{Eff})$ & & $6.2 \mathrm{E} 3$ & $\begin{array}{l}7 \mathrm{~d} \text { at } 2 \mathrm{E} 15 \mathrm{Cd} \text { shield } \\
\text { (Ref. 15) }\end{array}$ \\
\hline${ }^{199} \mathrm{Au}$ & $3.14 \mathrm{~d}$ & $2.08 \mathrm{E} 5$ & & & & \\
\hline $175 \mathrm{Hf}$ & $70 \cdot d$ & $1.07 \mathrm{E} 4$ & 390 & & $8 \mathrm{E} 3$ & $\operatorname{Max} 60 \mathrm{~d}$ at $8 \mathrm{E} 14$ \\
\hline $181_{\mathrm{Hf}}$ & $42.5 \mathrm{~d}$ & $1.69 \mathrm{E} 4$ & 12.6 & 40 & $6.4 \mathrm{E} 2$ & $90 \mathrm{~d}$ at $8 \mathrm{E} 14$ \\
\hline${ }_{111}^{3} \mathrm{H}$ & $\begin{array}{l}12.3 \mathrm{y} \\
2.83 \mathrm{~d}\end{array}$ & $\begin{array}{l}9.64 \mathrm{E} 3 \\
4.1 \mathrm{E} 5\end{array}$ & & & & \\
\hline $114 \mathrm{~m}$ In & $\begin{array}{l}2.83 \mathrm{~d} \\
49.5 \mathrm{~d}\end{array}$ & $\begin{array}{l}4.1 \mathrm{ES} \\
2.3 \mathrm{E} 4\end{array}$ & $7.7 \mathrm{R}$ & & $6.7 \mathrm{E} 2$ & $100 \mathrm{~d}$ at $8 \mathrm{E} 14$ \\
\hline $123 I$ & $13.2 \mathrm{~h}$ & $1.9 \mathrm{E} 6$ & & & & \\
\hline $125 \mathrm{I}$ & $60 \mathrm{~d}$ & $1.74 \mathrm{E} 4$ & & & & . \\
\hline${ }^{129} \mathrm{I}$ & $\begin{array}{l}1.57 \text { E7 y } \\
(\operatorname{Ref.4)}\end{array}$ & $1.7 \mathrm{E}-4$ & & & 1. $5 E-4$ & $86 \%(O R N L-4343)$ \\
\hline $130 \mathrm{I}$ & $12.3 \mathrm{~h}$ & $2.0 \mathrm{E} 6$ & 28 & 18 & $5.2 \mathrm{E} 3$ & $1 \mathrm{~d}$ at $2 \mathrm{E} 15$ \\
\hline${ }^{131} I$ & $8.05 \mathrm{~d}$ & $1.24 \mathrm{E} 5$ & & & $7.0 \mathrm{E} 4$ & ORNL -4039 \\
\hline $192 \mathrm{Ir}$ & $74 \mathrm{~d}$ & $9.2 \mathrm{E} 3$ & 9. $3 \mathrm{E} 2$ & 1. $1 E 3$ & $3.9 \mathrm{E} 3$ & $\operatorname{Max} 13 \mathrm{~d}$ at $8 \mathrm{E} 14$ \\
\hline $194 \mathrm{Ir}$ & $19.4 \mathrm{~h}$ & $8.3 \mathrm{E} 5$ & $1=0$ & & $1.6 \mathrm{E} 4$ & $2.5 \mathrm{~d}$ at $2 \mathrm{E} 15$ \\
\hline $55 \mathrm{Fe}$ & $2.7 \mathrm{y}$ & $2.4 \mathrm{E} 3$ & 2.2 & & $1.1 \mathrm{E} 2$ & 1 y at $8 \mathrm{E} 14$ \\
\hline${ }^{59} \mathrm{Fe}$ & $44.6 \mathrm{~d}$ & $5.0 \mathrm{E} 4$ & 1.1 & & $1.9 \mathrm{E} 2$ & $90 \mathrm{~d}$ at $8 \mathrm{E} 14$ \\
\hline${ }^{79} \mathrm{Kr}$ & $35 \mathrm{~h}$. & $1.13 \mathrm{E} 6$ & 4.9 & & $1.8 \mathrm{E} 4$ & $7 \mathrm{~d}$ at $2 \mathrm{E} 15$ \\
\hline $85_{\mathrm{Kr}}^{2}$ & $10.7 \mathrm{y}$ & $3.9 \mathrm{E} 2$ & & & $1.7 \mathrm{E} 2$ & $\begin{array}{l}44 \% \text { abundance } \\
(\mathrm{TM}-3558)\end{array}$ \\
\hline $137 \mathrm{La}$ & $6 E 4 y$ & $4 E-2$ & & & & \\
\hline${ }^{140} \mathrm{La}$ & $40.3 \mathrm{~h}$ & $5.5 \mathrm{E} 5$ & 8.9 & 2.7 & 1. $3 \mathrm{E} 3$ & $2.5 \mathrm{~d}$ at $2 \mathrm{E} 15$ \\
\hline $203 \mathrm{~Pb}$ & $52 \mathrm{~h}$ & 3.0E5 & & & & \\
\hline${ }^{197} \mathrm{Hg}$ & $64.1 \mathrm{~h}$ & $2.5 \mathrm{E} 5$ & $3.1 \mathrm{E} 3$ & & $1.7 \mathrm{E} 5$ & $\operatorname{Max} 63 \mathrm{~h}$ at $2 E 15$ \\
\hline $20{ }^{3} \mathrm{Hg}$ & $46.7 \mathrm{~d}$ & $1.4 \mathrm{E} 4$ & 4.9 & & $2.3 \mathrm{E} 2$ & $90 \mathrm{~d}$ at $8 \mathrm{E} 14$ \\
\hline $99 \mathrm{Mo}$ & $66.2 \mathrm{~h}$ & $4.8 \mathrm{E} 5$ & $0.15 \mathrm{R}$ & & 42 & $7 \mathrm{~d}$ at $2 \mathrm{E} 15$ \\
\hline
\end{tabular}


Table 1. Continued

\begin{tabular}{|c|c|c|c|c|c|c|}
\hline Nuclide & Half-Life & $\begin{array}{c}\text { Theoretical } \\
\text { Specific } \\
\text { Activity } \\
\text { (Ci/g) }\end{array}$ & $\begin{array}{l}\sigma_{A} \text { of } \\
\text { Target } \\
\text { Nuclide } \\
\text { (barns) }\end{array}$ & $\begin{array}{l}\sigma_{\mathrm{B}} \text { of } \\
\text { Radio- } \\
\text { nuclide } \\
\text { (barns) }\end{array}$ & $\begin{array}{c}\text { Practical } \\
\text { Specific } \\
\text { Activity } \\
(\mathrm{C} i / g)\end{array}$ & Remarks \\
\hline${ }^{99} \mathrm{Mo}$ & $66.2 \mathrm{~h}$ & $4.8 \mathrm{E} 5$ & & & $6.5 \mathrm{E} 4$ & FP, 7-d irradiation \\
\hline $147 \mathrm{Nd}$ & $11.0 \mathrm{~d}$ & $8.1 \mathrm{E} 4$ & & & $6.4 \mathrm{E} 3$ & $\mathrm{FP}, 28 \mathrm{~d}$ \\
\hline $237_{\mathrm{N}}$ & $2.14 \mathrm{E} 6 \mathrm{y}$ & $7.05 \mathrm{E}-4$ & & & & \\
\hline${ }^{59} \mathrm{Ni}$ & $8 \mathrm{E} 4 \mathrm{y}$ & $8 \mathrm{E}-2$ & 4.4 & 85 & $3.3 E-3$ & $\begin{array}{l}\text { Max } 200 \mathrm{~d} \text { at } 2 \mathrm{E} 15 \\
\text { (ORNL-4749) }\end{array}$ \\
\hline${ }^{63} \mathrm{Ni}$ & $\begin{array}{l}100 y \\
\text { (Ref. 16) }\end{array}$ & 57 & 15 & $\begin{array}{l}23 \\
(\operatorname{Ref} .16)\end{array}$ & 20 & 1 y at $8 \mathrm{E} 14$ \\
\hline${ }^{66} \mathrm{Ni}$ & $55 \mathrm{~h}$ & $8.6 \mathrm{E5}$ & $1.5(64)$ & $\sim 65(65)$ & 1.2 & $\begin{array}{l}5 \mathrm{~d} \text { at } 2 \mathrm{E} 15 \\
\text { Calc. from Ref. } 17\end{array}$ \\
\hline $92 \mathrm{~m} N \mathrm{~b}$ & $10.1 \mathrm{~d}$ & $1.40 \mathrm{E} 5$ & & & & \\
\hline${ }^{94} \mathrm{Nb}$ & $2.0 \mathrm{E} 4 \mathrm{y}$ & 0.18 & 1.1 & 11 & $5 E-3$ & $1 \mathrm{y}$ at $8 \mathrm{E} 14$ \\
\hline $95 \mathrm{Nb}$ & $35.1 \mathrm{~d}$ & $3.9 \mathrm{E} 4$ & & & & \\
\hline $1910 \mathrm{~s}$ & $15 \mathrm{~d}$ & $4.6 \mathrm{E} 4$ & 13 & & $8 \mathrm{E} 2$ & 28 d at $8 \mathrm{E} 14$ \\
\hline${ }^{109} \mathrm{Pd}$ & $13.5 \mathrm{~h}$ & $2.1 \mathrm{E} 6$ & $12 \mathrm{R}$ & & $2.6 \mathrm{E} 3$ & $1 \mathrm{~d}$ at $2 \mathrm{E} 15$ \\
\hline $32 \mathrm{P}$ & $14.3 \mathrm{~d}$ & $2.85 \mathrm{E} 5$ & & & $2.5 \mathrm{E} 5$ & ORNL-4196 \\
\hline${ }^{33} \mathrm{P}$ & $25 \mathrm{~d}$ & $1.6 \mathrm{E} 5$ & & & $3.2 \mathrm{E} 4$ & $20 \%(\mathrm{TM}-3414)$ \\
\hline${ }^{236} \mathrm{Pu}$ & $2.85 \mathrm{y}$ & $5.32 \mathrm{E} 2$ & & & & \\
\hline $238 \mathrm{Pu}$ & $87.5 \mathrm{y}$ & 17.2 & & & & \\
\hline${ }^{239} \mathrm{Pu}$ & $2.44 \mathrm{E} 4 \mathrm{y}$ & $6.14 \mathrm{E}-2$ & & & & \\
\hline $240 \mathrm{Pu}$ & $6.54 \mathrm{E} 3 \mathrm{y}$ & 0.229 & & & & \\
\hline $241 \mathrm{Pu}$ & $14.8 \mathrm{y}$ & 100 & & & & \\
\hline $242 \mathrm{Pu}$ & $3.87 \mathrm{E} 5 \mathrm{y}$ & $3.82 \mathrm{E}-3$ & & & & \\
\hline${ }^{42} \mathrm{~K}$ & $12.4 \mathrm{~h}$ & $6.0 \mathrm{E} 6$ & 1.3 & & $8 \mathrm{E} 2$ & $1 \mathrm{~d}$ at $2 \mathrm{E} 15$ \\
\hline $43_{\mathrm{K}}$ & $22.3 \mathrm{~h}$ & $3.3 \mathrm{E} 6$ & 20 & & & \\
\hline $142 \mathrm{Pr}$ & $19.2 \mathrm{~h}$ & $1.15 \mathrm{E} 6$ & 12 & 18 & $2.4 \mathrm{E} 3$ & $2.5 \mathrm{~d}$ at $2 \mathrm{E} 15$ \\
\hline $143 \mathrm{Pr}$ & $13.6 \mathrm{~d}$ & $6.7 \mathrm{E} 4$ & & & $4.5 \mathrm{E} 4$ & $\mathrm{FP}, 28 \mathrm{~d}$ \\
\hline $147 \mathrm{Pm}$ & $2.62 \mathrm{y}$ & $9.28 \mathrm{E} 2$ & & & & \\
\hline $148 \mathrm{mPm}$ & $42 \mathrm{~d}$ & $2.0 \mathrm{E} 4$ & 90 & $2.5 \mathrm{E} 4$ & 74 & $\operatorname{Max} 1.3 \mathrm{~d}$ at $2 \mathrm{E} 15$ \\
\hline
\end{tabular}


Tab.le 1. Continued

\begin{tabular}{|c|c|c|c|c|c|c|}
\hline Nuclide & Half-ífe & $\begin{array}{c}\text { Theoretical } \\
\text { Specific } \\
\text { Activity } \\
\text { (Ci/g) }\end{array}$ & $\begin{array}{l}\sigma_{A} \text { of } \\
\text { Target } \\
\text { Nuclide } \\
\text { (barns) }\end{array}$ & $\begin{array}{l}\sigma_{\mathrm{B}} \text { of } \\
\text { Radio- } \\
\text { nuclide } \\
\text { (barns) }\end{array}$ & $\begin{array}{c}\text { Practical } \\
\text { Specific } \\
\text { Activity } \\
(\mathrm{Ci} / \mathrm{g})\end{array}$ & Remarks \\
\hline${ }^{186} \mathrm{Re}$ & $\dot{91} \mathrm{~h}$ & $1.9 \mathrm{E} 5$ & 110 & & 1. $3 \mathrm{E} 4$ & $7 \mathrm{~d}$ at $2 \mathrm{E} 15$ \\
\hline $188 \mathrm{Re}$ & $16.9 \mathrm{~h}$ & $9.9 \mathrm{E} 5$ & 75 & & $1.2 \mathrm{E} 4$ & $2.5 \mathrm{~d}$ at $2 \mathrm{E} 15$ \\
\hline $102 \mathrm{Rh}$ & $206 \mathrm{~d}$ & $6.2 \mathrm{E} 3$ & & & & \\
\hline $83 \mathrm{Rb}$ & $86 \mathrm{~d}$ & $1.8 \mathrm{E} 4$ & & & & \\
\hline${ }^{84} \mathrm{Rb}$ & $34 d$ & $4.6 \mathrm{E} 4$ & & & & \\
\hline $86 \mathrm{Rb}$ & $18.7 \mathrm{~d}$ & $8.1 \mathrm{E} 4$ & $0.5 \mathrm{R}$ & & 49 & $28 \mathrm{~d}$ at $8 \mathrm{E} 14$ \\
\hline${ }^{97} \mathrm{Ru}$ & $2.9 \mathrm{~d}$. & $4.6 \mathrm{E} 5$ & $0.2 \mathrm{R}$ & & 60 & $9 \mathrm{~d}$ at $2 \mathrm{E} 15$ (TM-2910) \\
\hline $103_{\mathrm{Ru}}$ & $39.5 \mathrm{~d}$ & $3.2 \mathrm{E} 4$ & & & 4. $3 E 3$ & $\mathrm{FP}, 60 \mathrm{~d}$ \\
\hline $106_{\mathrm{Ru}}$ & $1.01 \mathrm{y}$ & $3.3 \mathrm{E} 3$ & & & 85 & $\mathrm{FP}, 1 \mathrm{y}$ \\
\hline $151_{\mathrm{Sm}}$ & $90 \mathrm{y}$ & 26 & & & & \\
\hline $153 \mathrm{Sm}$ & $46.7 \mathrm{~h}$ & $4.4 \mathrm{E} 5$ & $2=0$ & & $3.5 \mathrm{E} 4$ & $7 \mathrm{~d}$ at $2 \mathrm{E} 15$ \\
\hline $\begin{array}{l}46 \mathrm{Sc} \\
{ }^{47} \mathrm{Sc}\end{array}$ & $\begin{array}{l}83.8 \mathrm{~d} \\
3.4 \mathrm{~d}\end{array}$ & $\begin{array}{l}3.4 \mathrm{E} 4 \\
8.2 \mathrm{E} 5\end{array}$ & 25 & 8 & $5.5 \mathrm{E} 3$ & $180 \mathrm{~d}$ at $8 \mathrm{E} 14$ \\
\hline $\begin{array}{l}75 \mathrm{Se} \\
32 \mathrm{Si}\end{array}$ & $\begin{array}{r}120 \mathrm{~d} \\
\sim 5 \mathrm{E} 2 \mathrm{y}\end{array}$ & $\begin{array}{l}1.4 \mathrm{E} 4 \\
222\end{array}$ & 55 & . & $5.8 \mathrm{E} 3$ & $\operatorname{Max} 210 \mathrm{~d}$ at $8 \mathrm{E} 14$ \\
\hline $110 \mathrm{~m}_{\mathrm{Ag}}$ & $250 \mathrm{~d}$ & $4.7 \mathrm{E} 3$ & $\begin{array}{l}4.1 \\
(93 \text { tot. })\end{array}$ & 82 & $1.6 \mathrm{E} 2$ & Max 135 d at $8 \mathrm{E} 14$ \\
\hline $111 \mathrm{Ag}$ & $7.45 \mathrm{~d}$ & $1.6 \mathrm{E} 5$ & & & & \\
\hline${ }^{24} \mathrm{Na}$ & $15.0 \mathrm{~h}$ & $8.7 \mathrm{E} 6$ & 0.53 & & $5 \mathrm{E} 2$ & $1 \mathrm{~d}$ at $2 \mathrm{E} 15$ \\
\hline $85 \mathrm{Sr}$ & $64.5 \mathrm{~d}$ & $2.4 \mathrm{E} 4$ & 0.8 & & 73 & $90 \mathrm{~d}$ at $8 \mathrm{E} 14$ \\
\hline${ }^{89} \mathrm{Sr}$ & $50.5 \mathrm{~d}$ & $2.9 \mathrm{E} 4$ & & & $6.4 \mathrm{E} 3$ & $\mathrm{FP}, 90 \mathrm{~d}$ \\
\hline $\begin{array}{l}90 \mathrm{Sr} \\
35 \mathrm{~S}\end{array}$ & $\begin{array}{l}28.5 \mathrm{y} \\
88 \mathrm{~d}\end{array}$ & $\begin{array}{l}140 \\
4.2 E 4\end{array}$ & & & 77 & $55 \%($ ORNL-IIC-36) \\
\hline $182 \mathrm{Ta}$ & $115 \mathrm{~d}$ & $6.2 \mathrm{E} 3$ & $2: \mathrm{R}$ & $8 \mathrm{E} 3$ & 16 & Max $14 \mathrm{~d}$ at $8 \mathrm{E} 14$ \\
\hline $\begin{array}{r}95 \mathrm{~m}_{\mathrm{Tc}} \\
{ }^{99} \mathrm{Tc}\end{array}$ & $\begin{array}{l}61 \mathrm{~d} \\
2.13 \mathrm{E} 5 \mathrm{y}\end{array}$ & $\begin{array}{l}2.3 \mathrm{E} 4 \\
1.70 \mathrm{E}-2\end{array}$ & & & & \\
\hline $12 \mathrm{~lm}_{\mathrm{Te}}$ & $154 \mathrm{~d}$ & $7.0 \mathrm{E} 3$ & 0.34 & & 30 & $1 \mathrm{y}$ at $8 \mathrm{E} 14$ \\
\hline $1.23 \mathrm{~m}_{\mathrm{Te}}$ & $120 \mathrm{~d}$ & $8.8 \mathrm{E} 3$ & 0.3 & $5 \mathrm{E} 3$ & $\sim 1$ & $3 \mathrm{~d}$ at $1 \mathrm{E} 15$ (TM-3708) \\
\hline
\end{tabular}


Table 1. Continued

\begin{tabular}{|c|c|c|c|c|c|c|}
\hline Nuclide & Half-Life & $\begin{array}{c}\text { Theoretical } \\
\text { Specific } \\
\text { Activity } \\
(\mathrm{C} i / g)\end{array}$ & $\begin{array}{l}\sigma_{\text {A }} \text { of } \\
\text { Target } \\
\text { Nuclide } \\
\text { (barns) }\end{array}$ & $\begin{array}{l}\sigma_{\mathrm{B}} \text { of } \\
\text { Radio- } \\
\text { nuclide } \\
\text { (barns). }\end{array}$ & $\begin{array}{l}\text { Practical } \\
\text { Specific } \\
\text { Activity } \\
\text { (Ci/g) }\end{array}$ & Remarks \\
\hline $125 \mathrm{~m}_{\mathrm{Te}}$ & $58 \mathrm{~d}$ & $1.8 \mathrm{E} 4$ & & & & \\
\hline $132 \mathrm{Te}$ & $78 \mathrm{~h}$ & $3.0 \mathrm{E} 5$ & & & 1. $3 \mathrm{E} 5$ & $\mathrm{FP}, 7 \mathrm{~d}$ \\
\hline $156 \mathrm{~Tb}$ & $5.4 \mathrm{~d}$ & $1.6 \mathrm{E} 5$ & & & & \\
\hline $204 \mathrm{~T} 1$ & $3.78 \mathrm{y}$ & $4.6 \mathrm{E} 2$ & 11 & 22 & 85 & $1 \mathrm{y}$ at $8 \mathrm{E} 14(\mathrm{ORNL}-4306)$ \\
\hline${ }^{229} \mathrm{Th}$ & 7. 34E3 y & $0: 213$ & & & & \\
\hline $230 \mathrm{Th}$ & $7.7 \mathrm{E} 4 \mathrm{y}$ & $2.02 \mathrm{E}-2$ & & & & \\
\hline $170 \mathrm{Tm}$ & $128 \mathrm{~d}$ & $6.0 \mathrm{E} 3$ & $1.15 \mathrm{R}$ & 92 & $2.1 \mathrm{E} 3$ & Max $100 \mathrm{~d}$ at $8 \mathrm{E} 14$ \\
\hline${ }^{171} \mathrm{Tm}$ & $7 \mathrm{E}_{2} \mathrm{~d}$ & $1.1 \mathrm{E} 3$ & & & & \\
\hline $113 \mathrm{Sn}$ & $115 \mathrm{~d}$ & $1.00 \mathrm{E} 4$ & $0.7 \mathrm{R}$ & & 51 & $180 \mathrm{~d}$ at $8 \mathrm{E} 14$ \\
\hline $181_{W}$ & $121.5 \mathrm{~d}$ & $5.9 \mathrm{E} 3$ & 10 & & $5 \mathrm{E} 2$ & $180 \mathrm{~d}$ at $8 \mathrm{E} 14$ \\
\hline $185 \mathrm{~W}$ & $75 \mathrm{~d}$ & $9.4 \mathrm{E3}$ & 1.8 & & $1.0 \mathrm{E} 2$ & $180 \mathrm{~d}$ at $8 \mathrm{E} 14$ \\
\hline $187 \mathrm{~W}$ & $24 \mathrm{~h}$ & $7.0 \mathrm{E} 5$ & 33 & 64 & $4.7 \mathrm{E} 3$ & $\begin{array}{l}2.5 \mathrm{~d} \text { at } 2 \mathrm{E} 15 \\
(\mathrm{CF}-65-12-30)\end{array}$ \\
\hline $188 \mathrm{~W}$ & $69 \mathrm{~d}$ & $1.01 \mathrm{E} 4$ & $33(186)$ & $64(187)$ & 12 & $140 \mathrm{~d}$ at $8 \mathrm{E} 14$ \\
\hline $23^{3} \mathrm{U}$ & $1.58 \mathrm{E} 5 \mathrm{y}$ & $9.68 \mathrm{E}-3$ & & & & \\
\hline $234 \mathrm{U}$ & $2 . \angle 8 \mathrm{E} 5 \mathrm{y}$ & $6.16 E-3$ & & & & \\
\hline $235_{U}$ & $7.04 \mathrm{E} 8 \mathrm{y}$ & $2.16 \mathrm{E}-6$ & & & & \\
\hline $236 \mathrm{U}$ & $2 . \angle 0 E 7 y$ & $6.31 E-5$ & & & & \\
\hline $238 \mathrm{U}$ & $4 . \angle 7 \mathrm{E} 9$ y & $3.36 \mathrm{E}-7$ & & & & \\
\hline${ }^{48} \mathrm{~V}$ & $16.0 \mathrm{~d}$ & $1.7 \mathrm{E} 5$ & & & & \\
\hline $129 \mathrm{~m}_{\mathrm{Xe}}$ & $8 \dot{c}$ & $1.26 \mathrm{E} 5$ & $6.2\left({ }^{127} I\right)$ & $0.43\left({ }^{128} \mathrm{Xe}\right)$ & 40 & 120 d at $8 \mathrm{E} 14$ \\
\hline $13 \operatorname{lm}_{\mathrm{Xe}}$ & $12.0 \mathrm{~d}$ & 8. 3E4 & $28\left({ }^{129} \mathrm{I}\right)$ & $0.34\left({ }^{130} \mathrm{Xe}\right)$ & 30 & $120 \mathrm{~d}$ at $8 \mathrm{E} 14$ \\
\hline $133 \mathrm{Xe}$ & $5.29 \mathrm{~d}$ & $1.85 \mathrm{E} 5$ & & & $1.5 \mathrm{E} 4$ & FP, $8 \%$ abundance \\
\hline${ }^{86} \mathrm{Y}$ & $14.7 \mathrm{~h}$ & $2.5 \mathrm{E} 6$ & & & & 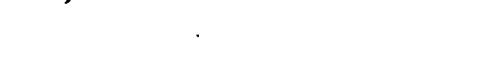 \\
\hline${ }^{87} \mathrm{Y}$ & $80 \mathrm{~h}$ & $4.5 \mathrm{E} 5$ & & & & \\
\hline $88 \mathrm{Y}$ & $107 \mathrm{~d}$ & $1.4 \mathrm{E} 4$ & & & & \\
\hline${ }^{90} \mathrm{Y}$ & $64.0 \mathrm{~h}$ & $5.4 \mathrm{E5}$ & & & & \\
\hline $91_{Y}$ & $59 \mathrm{~d}$ & $2.4 \mathrm{E} 4$ & & & $2.3 \mathrm{E} 4$ & $\begin{array}{l}\text { FP, } \sim 95 \% \text { abundance } \\
\text { (ORNL-4343) }\end{array}$ \\
\hline
\end{tabular}


Table 1. Continued

\begin{tabular}{|c|c|c|c|c|c|c|}
\hline Nuclide & Half-Life & $\begin{array}{c}\text { Theoretical } \\
\text { Specific } \\
\text { Activity } \\
(\mathrm{C} i / g)\end{array}$ & $\begin{array}{l}\sigma_{A} \text { of } \\
\text { Target } \\
\text { Nuclide } \\
\text { (barns) }\end{array}$ & $\begin{array}{l}\sigma_{\mathrm{B}} \text { of } \\
\text { Radio- } \\
\text { nuclide } \\
\text { (barns) }\end{array}$ & $\begin{array}{l}\text { Practical } \\
\text { Specific } \\
\text { Activity } \\
(\mathrm{Ci} / \mathrm{g})\end{array}$ & Remarks \\
\hline $\begin{array}{r}65 \mathrm{Zn} \\
69 \mathrm{~m}_{\mathrm{Zn}} \\
95 \mathrm{Zr}\end{array}$ & $\begin{array}{l}244 \mathrm{~d} \\
13.8 \mathrm{~h} \\
65 \mathrm{~d}\end{array}$ & $\begin{array}{l}8.2 E 3 \\
3.3 E 6 \\
2.1 E 4\end{array}$ & 0.7 & & $\begin{array}{l}90 \\
3 E 3\end{array}$ & $\begin{array}{l}1 \text { y at } 8 \mathrm{E} 14 \\
\text { FP, } 90 \mathrm{~d}\end{array}$ \\
\hline
\end{tabular}

$a{ }^{\prime}$ " following the cross-section value indicates that resonance activation is significant. 
Practical specific activities are those actually realized at ORNL, or calculated for readily obtainable conditions. For neutron-capture products, listed cross sections are $\sigma_{A}$, the thermal cross section for formation of a particular nuclide, and $\sigma_{B}$ for its destruction, unless otherwise noted. The values are compatible with those compiled ${ }^{6}$ for the 1972 Chart of the Nuclides, except for a few determined locally, or found in recent literature. The yields were calculated as usual, ${ }^{7}$ and the specific activities are actual, taking into account any transmutations during irradiation. Isotopically pure targets were assumed, and flux values were those typical of the ORNL High Flux Isotope Reactor (HFIR), 2El5 for a week or less, and $8 \mathrm{E} 14$ for longer irradiations. "Max" indicates time of maximum yield of the product at the given flux. Unless dictated otherwise, desirable bombardments of 1-2 half-1ives (50-75\% "saturation") were chosen. References to Oak Ridge National Laboratory documents (ORNL, TM, IIC, and CF) are given under "remarks," and call-outs are listed for outside publications. Values were compared with an earlier table, ${ }^{8}$ which gave curies per gram of target for a variety of fluxes.

Because of the uncertainties in most cross sections, corrections for effects causing errors of $10 \%$ or less were ignored if they made calculations more tedious. Resonance activation was not included, but its occurrence is indicated by " $R$ " in cases where it would be comparable to thermal activation of a thin sample in a typical reactor spectrum. Self-shadowing was also not considered, although its effect can be quite large. These two effects are qualitatively compensating.

For fission products (FP), allowance was made for isotopic composition, as calculated from fission yields. ${ }^{9}$ Secondary neutron capture by fission products was ignored. In some cases, actual compositions found for ORNL products are noted.

\section{RADIOISOTOPE CHARACTERISTICS}

Up-to-date values for decay parameters of 17 radionuclides, now known to be significantly different from those given in the Isotope User's Guide, are listed in Table 2. It must be noted that most of the nuclides have other properties listed in the Guide which remain unchanged. Nuclear Data is the source of most of the adoptions and should be consulted for details. Intensities of $\mathrm{K} \times$ rays are included here for several nuclides for which those are the most significant radiations. Important recent developments in other areas discussed in the Guide include a surveylo of the radioactivity standards situation by a National Research Council panel, continuing contributions by the National Bureau of Standards, 11,12 revisions (now in progress) of an NCRP handbook ${ }^{13}$ and the Users' Guides for Radioactivity Standards, and a review of ORNL assay methods. 14 It should be noted that many radionuclides decay to active daughters, whose disintegration rates can be less than, equal to, or greater than those of the parents, depending on the relative values of the half-lives, and the time of production or separation. ${ }^{7}$ 
Table 2. Changes in Radionuclide Properties

\begin{tabular}{|c|c|c|c|}
\hline Nuclide & Half-Iife & & Photons ${ }^{a}$ \\
\hline $133 \mathrm{Ba}$ & & & Cs $\mathrm{K} \times$ rays $(118)$ \\
\hline $140 \mathrm{Ba}$ & 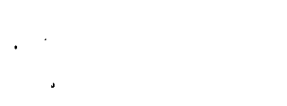 & & $\begin{array}{l}0.030(14) \\
0.537(24)\end{array}$ \\
\hline${ }^{10} \mathrm{Be}$ & $1.6 \mathrm{E} 6 \mathrm{y}$ (Ref. & 18) & \\
\hline $\mathrm{Cd}-10.9 \mathrm{Ag}$ & & & $\begin{array}{l}\mathrm{Ag} \mathrm{K} \times \text { rays }(100) \\
0.088(3.8)\end{array}$ \\
\hline${ }^{67} \mathrm{Cu}$ & & & $0.185(47)$ \\
\hline${ }^{155} \mathrm{Eu}$ & $4.8 \mathrm{y}$ & & \\
\hline${ }^{67} \mathrm{Ga}$ & & & $\begin{array}{l}\mathrm{Zn} \mathrm{K} \times \text { rays }(25) \\
0.093(42) \\
0.185(24) \\
0.300(17) \\
0.394(4.6)\end{array}$ \\
\hline${ }^{124} \mathrm{I}$ & . & & $\begin{array}{l}\mathrm{Te} K \times \text { rays }(57) \\
\gamma^{ \pm}(46) \\
0.603(62) \\
0.723(10) \\
1.69(11) \\
\text { Others }\end{array}$ \\
\hline${ }^{125} \mathrm{I}$ & & & $\mathrm{Te} K \times$ rays $(136)$ \\
\hline${ }^{129} \mathrm{I}$ & & & $\mathrm{Xe} K \times$ rays $(68)$ \\
\hline${ }^{55} \mathrm{Fe}$ & & & Mn $Y . x$ rays $(2 h)$ \\
\hline${ }^{79} \mathrm{Kr}$ & & & Cr $\mathrm{K} \times$ rays $(51)$ \\
\hline $191 \mathrm{mos}$ & & & Os $\mathrm{x}$ rays \\
\hline $193 \mathrm{~m}_{\mathrm{Pt}}$ & $\sim 4.0 \mathrm{~d}$ & & \\
\hline $\begin{array}{c}195 \mathrm{~m}_{\mathrm{Pt}} \\
.\end{array}$ & $4.0 \mathrm{~d}$ & & $\begin{array}{l}0.099(12) \\
0.129(3) \\
\text { Pt x rays }\end{array}$ \\
\hline $108 \mathrm{~m}_{\mathrm{Ag}}$ & $\sim 127 \mathrm{y}$ & & $\begin{array}{l}0.434(90) \\
0.614(91) \\
0.223(91) \\
\mathrm{Pd}, \mathrm{Ag} \times \text { rays }\end{array}$ \\
\hline${ }^{181} \mathrm{~W}$ & & & $\mathrm{Ta} \mathrm{K} \times$ rays $(60)$ \\
\hline
\end{tabular}

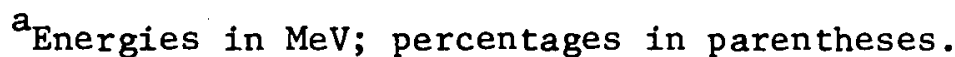




\section{CONCLUSION}

The values for half-lives, cross sections, and other characteristics have been selected to assure reasonable accuracy in applications in such fields as biomedicine, analysis, and source technology. Questions about the values selected and about ORNL work should be directed to the author, and additional information would be gratefully accepted.

\section{REFERENCES}

1. Research Materials, Enriched Isotopes, Radioisotopes, Special Preparations, Oak Ridge National Laboratory (1973).

2. F. E. McKinney, S. A. Reynolds, and P. S. Baker, Isotope User's Guide, ORNL-IIC-19, Oak Ridge National Laboratory (1969).

3. M. J. Martin and P. H. Blichert-Toft, Nuclear Data A8, 1 (1970).

4. J. F. Emery, S. A. Reynolds, E. I. Wyatt, and G. I. Gleason, Nucl. Sci. Eng. $\underline{48}, 319$ (1972).

5. G. Goldstein and S. A. Reynolds, Nuclear Data Al, 435 (1966).

6. N. E. Holden, Knolls Atomic Power Laboratory, personal communication, 1972 .

7. G. Friedlander, J. W. Kennedy, and J. M. Miller, Nuclear and Radiochemistry, 2nd ed., Wiley, New York, 1964.

8. T. C. Whitson and C.W. Friend, Reactor Yield Calculations for 81 Radioisotopes Produced by $(n, \gamma)$ Reactions at Fluxes of $10^{7}$ to $10^{16}$ $\mathrm{n} / \mathrm{cm}^{2}$.sec for Irradiation Times of 30 Minutes to One Year, ORNLIIC-8, Oak Ridge National Laboratory (1966).

9. M. E. Meek and B. F. Rider, Compilation of Fission Product yiezds, NEDO-12154, Vallecitos Nuclear Center (1972).

10. National Uses and Needs for Standard Radioactive Materials, National Academy of Sciences, Washington (1970).

11. Standard Roferonce Matorial.s, NBS SP 260 (annual), Government Printing Office, Washington.

12. Calibration and Test Services of the NBS, NBS SP 250 (1970 plus revisions), Government Printing office, Washington.

13. A Manual of Radioactivity Procedures, NCRP Rpt. 28 (NBS Handbook 80), Government Printing Office, Washington (1961). 
14. S. A. Reynolds, Some Radioisotope Assay Methods, ORNL-4788, 0ak Ridge National Laboratory (1973).

15. R: E. Lewis, Nucl. Appl. 2, 33 (1966).

16. I. L. Barnes, S. B. Garfinke1, and W. B. Mann, Int. J. App Z: Radiat. Isotop. 22, 777 (1971).

17. J. J. Pinajian, J. Inorg. Nucl. Chem. 31, 1241 (1969).

18. E. M. McMillan, Phys. Rev. C6, 2296 (1972). 
INTERNAL DISTRIBUTION

1. P. S. Baker

2. E. E. Beauchamp

3. G. E. Boyd

4. T: A. Butler

5. F. N. Case

6. J. H. Cooper

7. J. A. Cox

8. F. L. Culler

9. W. C. Davis

10. F. F. Dyer

11. J. F. Emery

12. J. H. Gillette

13. H. R. Gwinn

14. R, F, Hibbs

15. D. J. Horen

16. E. H. Kobisk

17. E. Lamb

18. L. 0. Love

19. W. S. Lyon

20. H. A. Parker

21. J. J. Pinajian

22. M. E. Pruitt
23. M. E. Ramsey

24. J. E. Ratledge

25-49. S. A. Reynolds

50. R. A. Robinson

51. A. F. Rupp

52. R. W. Schaich

53. W. D. Shults

54. A. H. Snell

55. M. R. Skidmore

56. H. F. Stringfield

57. J. R. Totter

58. D. B. Trauger

59. A. M. Weinberg

60. J. C. White

61. E. I. Wyatt

62. A. Zucker

63-64. Central Research Library

65. Document Reference Section

66. ORNL Patent Office

67-70. Laboratory Records Department

71. Laboratory Records - RC

\section{EXTERNAL DISTRIBUTION}

72. P. Alexander, NISC, Washington, DC.

73. G. A. Andrews, ORAU, Medical Division, Oak Ridge, Tenn.

74. D. S. Ballantine, AEC, Washington, DC.

75. E. J. Baratta, FUA, Winchester, Mass.

76. N. F. Barr, AEC, Washington, DC.

77. G. L. Borsheim, ARHCO, Richland, Washington.

78. J. C. Brantley, New England Nuclear Corporation, Boston, Mass.

79. R. L. Butenhoff, AEC, Washington, DC.

80. T. D. Chikalla, PNL, Richland, Washington.

81. G. R. Choppin, Florida State University, Tallahassee, Fla.

82. D. F. Cupe, AEC Site Representative, ORNL.

83. J. A. Corbett, Westinghouse, Madison, Pennsylvania.

84. A. Dahl, BRH, Rockville, Maryland.

85. J. C. Dempsey, AEC, Washington, DC.

86. B. J. Dropesky, LASL, Los Alamos, New Mexico.

87. E. H. Eisenhower, NBS, Washington, DC.

88. E. E. Fowler, AEC, Washington, DC.

89. G. I. Gleason, ORAU, Oak Ridge, Tenn.

90. T. D. Goldstein, AEC, Washington, DC.

91. A. Gottschalk, Argonne Cancer Research Hospital, Chicago.

92. F. D. Haines, AEC, Washington, DC.

93. W. Hauser, St. Mary's Hospital, Montreal, Province of Quebec.

94. R. L. Heath, Allied Nuclear, Idaho Falls, Idaho.

95. W. D. Holloman, AEC, Washington, DC

96. J. W. Irvine, MIT (consultant) 
97. J. H. Jarrett, PNL, Richland, Washington.

98. D. K. Jones, AEC, Richland, Washington.

99. B. Kahn, EPA, Cincinnati, Ohio.

100. R. C. Koch, International Chemical Nuclear, Pasadena, California

101. W. R. Konneker, Mallinckrodt, St. Louis, Mo.

102. J. Lawrence, Lawrence Berkeley Laboratory, Berkeley, California.

103. R. E. Lewis, GE, Pleasanton, California.

104. J. E. Machurek, AEC, Washington, DC .

105. J. N. Maddox, AEC, Washington, DC .

106. J. C. Malaro, AEC, Washington, DC.

107. B. Manowitz, BNL, Upton, New York.

108. L. A. Miller, AEC, Washington, DC.

109. W. E. Mott, AEC, Washington, DC.

110. H. A. O'Brien, LASL, Los Alamos, New Mexico.

111. M. J. Oestmann, AEC, Washington, DC.

112. J. A. Prestele, Consolidated Edison, New York, New York.

113. G. J. Rotariu, AEC, Washington, DC.

114. W. D. Sandberg, AEC, Aiken, South Carolina

115. C. Seidel, New England Nuclear, N. Billerica, Mass.

116. R. W. Shivers, AEC, Washington, DC.

117. J. Silverman, University of Maryland, College Park, Maryland.

118. F.J. Skozen (Krizek), Argonne Cancer Research Hospital, Chicago

119. Robert E. Smith, ARHCO, Richland, Washington.

120. R. F. Smith, Union Carbide, Paducah, Kentucky.

121. L. G. Stang, Jr., BNL, New York.

122. L. E. Steele, NRL, Washington, DC.

123. D. H. Turno, SRL, Aiken, South Carolina.

124. A. R. Van Dyken, AEC, Washington, DC .

125. K. L. Voigt, ANS, Hinsdale, Illinois.

126. Research and Technical Support Division, ORO

127-128. Technical Information Center. 